\title{
SEQUENTIAL RESPONSE BIAS IN RANDOMIZED RESPONSE SEQUENCES: A COMPUTER SIMULATION
}

\author{
S. WIEGERSMA * \\ University of Utrecht, The Netherlands
}

Accepted September 1982

\begin{abstract}
In this study sequential bias in randomized response sequences is simulated by computer. The computer model assumes that response bias is composed of two components. The first is a repetition avoidance tendency, which is simulated by a memory search through the seven most recently produced responses, and the second is a repetition tendency for the two most recently produced responses. This repetition tendency is assumed to be related to the better availability of recently produced responses. Both processes together give a reasonable approximation of human subjects' data.

The results can be explained by the assumption that subjects try to control perseveration tendencies by using a comparison-based memory search mechanism.
\end{abstract}

\section{Introduction}

It has been frequently observed that human subjects, when asked to produce a random sequence of responses chosen from a fixed set of responses, show various types of response tendencies. Several theoretical explanations can be forwarded to account for the observed phenomena.

A strength theory, for example, predicts that the recently produced responses are produced again, because they are assumed to be strongly available in memory. The strength theory, therefore, predicts the occurrence of more repetitions than expected in a true random sequence, but in practice an excess of repetitions is not a dominant phenomenon in such sequences. Instead, repetition avoidance tendencies have been found to exist to at least the sixth order of dependency (Wagenaar

* Author's address: S. Wiegersma, University of Utrecht, Dept. of Psychology, Varkenmarkt 2, 3511 BZ Utrecht. The Netherlands.

0001-6918/82/0000-0000/\$0.275 @ 1982 North-Holland 
1970). This means, that for a particular response, $R(n)$ repetitions of the previous responses $R(n-1), R(n-2), R(n-3), R(n-4), R(n-5)$ and $R(n-6)$ occur less frequently than expected from a random process. A possible explanation for repetition avoidance is an underlying memory search mechanism which depends on a comparison process rather than on the strengths of the items in memory. This would enable the subject to select more distant (weaker) responses rather than recent (stronger) ones. In the present paper a model of this search process is proposed which assumes a memory of recently produced responses using seven memory places. A retention function operates on the responses in memory, giving a probability of retention for each of the seven previous responses: the more recent the response the higher the probability that it is still available in memory. The system arbitrarily selects a probe item from the set of admissible responses; when the probe matches one of the items in memory it is rejected as a response. A new probe is randomly selected for comparison with the memory items, etc., until a response which does not match any of the memory items is produced. Such a system will produce series in which repetitions of all six orders are avoided to some extent. The plan of this study is to compare sequences produced by human subjects with the sequences generated by the simulation model.

\section{The experiment}

\section{Method}

Generation of sequences by computer

The system was tested for two linear retention functions. The assumption of linearity is based on an experiment of Wagenaar (1972). in which the recall of previously produced responses was studied. In this simulation we are not interested in the degree to which previous items can be recalled, but in how well they are retained and available for a search process. However, since there is no information about the retention function in a running memory setting, it is assumed that the linear recall function is a good approximation of this function. The two retention functions studied give these probabilities for responses which have been produced one to seven trials ago:

(a) $0.90,0.80,0.70,0.60,0.50,0.40,0.30$ (condition 0.90 ), and

(b) $0.70,0.60,0.50,0.40,0.30,0.20,0.10$ (condition 0.70 ).

With maximal forgetting (a retention function of $0.00,0.00$, etc.), the system accepts all 
responses generated. A test showed that no sequential dependencies occurred in series generated in this way.

Four series of 1000 numbers were generated for each retention function. $\phi$-values measuring the use of repetitions for six orders of dependency were calculated for all series. The computation of $\phi$ is as described in Wagenaar (1970), and can be summarized as follows. Expected frequencies of engrams $\left(e_{1} \ldots e_{n}\right)$ are estimated on the assumption of independence of observed frequencies by the formula:

$f\left(e_{1} e_{2} \ldots e_{n-1} e_{n}\right)=\frac{f\left(e_{1} e_{2} \ldots e_{n-1}\right) \times f\left(e_{2} \ldots e_{n-1} e_{n}\right)}{f\left(e_{2} \ldots e_{n-1}\right)}$

Observed frequencies of repetitive engrams $\left(e_{1}=e_{n}\right)$ and non-repetitive engrams $\left(e_{1} \neq\right.$ $\left.e_{n}\right)$ are then summarized to separate sums. The same is done for expected frequencies. Then, the observed frequencies $\left(O_{i}\right)$ of repetitions and non-repetitions are compared with the predicted frequencies $\left(E_{i}\right)$ by:

$\chi^{2}=\sum_{i} \frac{\left(O_{i}-E_{i}\right)^{2}}{E_{i}}$

The coefficient of randomness is given by:

$\phi=\sqrt{x^{2} / N}$

in which $N$ is the number of responses in the series. $\phi$ may vary between -1 and +1 . Negative values indicate less repetitions, positive values more repetitions than expected by chance.

\section{Data generation by human subjects}

18 undergraduate psychology students called out number series in which four alternatives (numbers 1-4) or eight alternatives (numbers 1-8) had to be used under three production rate conditions: 46,66 and 86 responses/minute. The six task conditions were presented to the $S \mathrm{~s}$ in a counterbalanced order, so that each condition occurred three times in every presentation position. Production rate was paced by the click of a metronome. The $S$ was instructed to produce a response simultaneously with each click of the metronome. The instruction to the $S \mathrm{~s}$ was as follows:

"You are asked to produce a number sequence. You have to speak the numbers in a rate given by this metronome. On each tick you have to produce one number. You are not allowed to use systems in producing the sequence. The only thing you have to strive for is to use all numbers equally of ten in the long run. Once you have started you have to go on until I tell you to stop. You have to use numbers from a set of admissible numbers, that will be indicated before the start of a sequence." 
Results

Eight response alternatives

Fig. 1 gives the mean $\phi$-values of the human $S$ s and of the computer-generated sequences for the case of eight response alternatives. It appears that neither of the computer-generated curves gives a good approximation to human performance. While the computer generates data which are highly linearly dependent on order, the subject-generated curve is clearly curvi-linear. However, condition 0.90 gives a good approximation for the third to sixth order of dependency. It must be concluded that the search model is insufficient to reproduce the results of the human $S$ s.

\section{The lower order dependencies}

Fig. 1 showed that human $S$ s produce more lower order repetitions than predicted by the model. This suggests that, in addition to a tendency to avoid repetitions of the seven most recently produced responses, there is a kind of 'repetition tendency' of only the last responses.

As an addition to the search model an extra repetition tendency was implemented. While generating the next response it was first determined whether the last response would be repeated. If so, this response was accepted for production. If not, it was determined whether the second to the last response would be repeated. From the experimental data it was concluded that repetition probabilities of 0.04 and 0.01 for the
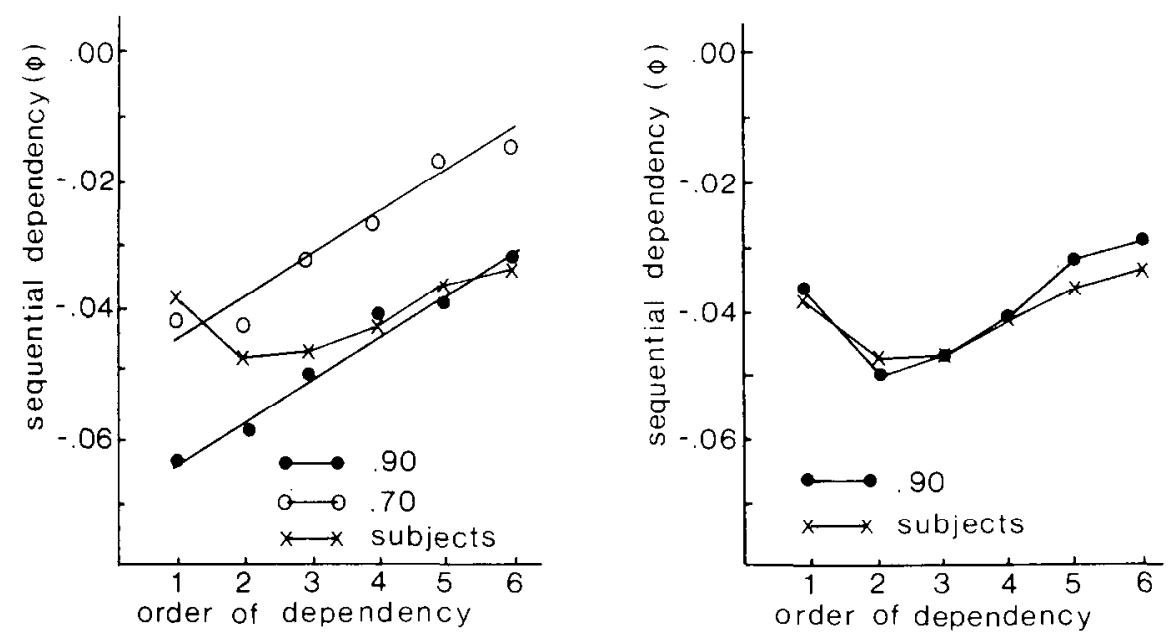

Fig. 1. Mean sequential dependencies for simulated series using two different retention functions, and for human subjects; eight alternatives.

Fig. 2. Mean sequential dependencies for simulated series with a retention function (0.90) and repetition tendencies $(0.04,0.01)$ in the lower orders, and for human subjects; eight alternatives. 
last and second to the last response would be good choices for these extra repetition tendencies. As shown by fig. 2 the model describes the observed result for eight response alternatives quite well.

\section{A test of the model}

A test of the model can be obtained by applying it to the other experimental condition of four response alternatives. The scores of the human $S$ s were again averaged over the three response rate conditions. The version best fitting the case of eight alternatives (condition 0.90 ; repetition tendencies: $0.04,0.01$ ) was used for the test. Nothing was changed in the program.

Fig. 3 gives the results of the simulation and of the $S \mathrm{~s}$ for four alternatives. The fit with the results of the $S \mathrm{~s}$ is tested with $t$-tests for one sample (Hays 1963: 305) for all six orders. There are two significant differences: for the first order $(t=-2.90, d f=53$, $p<0.01)$, and for the third order $(t=2.22, d f=53, p<0.05)$. It seems that the higher orders can be explained reasonably well by assuming a retention function which is independent of the number of alternatives. The repetition probability for the first order, however, is too high for the case of four alternatives. This is not in line with the idea of an autonomous repetition tendency which is independent of number of alternatives.

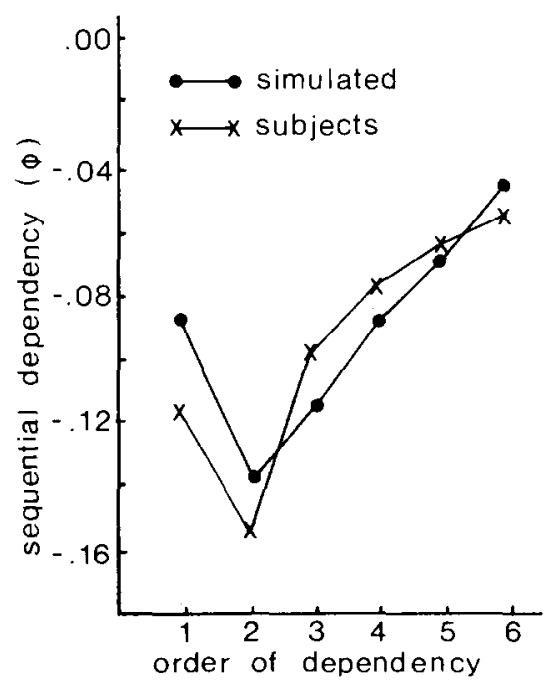

Fig. 3. Mean sequential dependencies for simulated series with a retention function $(0.90)$ and repetition tendencies $(0.04,0.01)$ in the lower orders, and for human subjects; four alternatives. 


\section{Discussion}

Repetition avoidance in sequential response production could not be sufficiently simulated by postulating that a memory search mechanism, using the decaying information about the seven last responses determines such behavior. This proposed repetition avoidance mechanism can explain only a main component in repetition avoidance. A second process, inclined to repetition, is needed to explain a component in the first and second order of dependency. However, such a repetition tendency is not constant over different set sizes. It is stronger for eight alternatives than for four alternatives.

The model fits in the general framework of a control theory which assumes a duality in the determination of response tendencies in the production of random sequences. On the one hand, there are tendencies to produce responses which come to mind because of their availability or memory strength, like 'perseverations' and stereotyped orders. On the other hand, subjects try to avoid such tendencies by the use of control processes (Wiegersma 1982).

The lower orders of response bias have been found to be different from the higher orders in some other studies. Kuhl and Schönpflug (1974) found an effect of instruction on the first order but not on the second and third order of dependency. Truijens et al. (1976) found that the effects of amphetamine and barbiturate were different for the three lower orders, but they did not differ for the higher orders. Such findings suggest that response bias in the lower orders is to some degree under attentional control, while the higher orders may be controlled rather automatically requiring no attention.

The two components of response bias remind us of two components found in short-term memory experiments, i.e. a primary and secondary component. The most recent items in such experiments are often recalled most easily. However, in production experiments the recency component is not represented by better recall, but by a repetition tendency, while the secondary component appears to be related to repetition avoidance. While in recall tasks both components add up to a better recall of recent items, they seem to have opposite effects in production. This suggests that each component reflects a different system of representation or retrieval of previous responses, each of which can affect behavior in a quite different way.

There are two general ways in which the duality in memory studies 
can be explained: by a dual-trace, or by a dual-retrieval theory. The dual-trace theory pedicts that previous responses are stored in two ways: at a verbal-categorical level (six or seven recent responses) and at a precategorical, e.g. acoustical level (the last few responses) (Crowder and Morton 1969). A dual-retrieval theory might be an alternative to the dual-trace explanation (Tulving 1968). The recency effect could be brought about by retrieval by means of positional cues associated to the most recent responses (Sanders 1975). The lower order repetition effects would then be related to the use of such positional cues, while repetition avoidance would be related to categorical cues. This clearly implies different strategies of the subject with respect to the use of these two types of retrieval cues. More experimentation is required before it can be decided whether a dual-trace or a dual-retrieval mechanism underlies the duality of sequential response bias.

The control theory has some special implications, some of which are briefly mentioned below. The extent to which response bias occurs is commonly described in terms of departures from randomness. For example, the effect of a simultaneous second task is that stereotyped responding increases beyond chance, e.g. the use of counting in number series (Evand and Graham 1980) whereas repetition avoidance may get less strong and approach randomness (Wagenaar 1972). Rather than concluding that the effects are opposite in terms of randomness (Evans and Graham 1980), one might conclude that both results reflect a decreased control of strength-determined tendencies in the double task conditions. Another application of the control theory concerns aphasia. Aphasic patients have problems in finding the words to express their thoughts. At the same time strength-dependent processes such as frequency effects and association seem to be intact. With respect to the randomization task one would predict that the memory search mechanism would be defect while the lower order repetition tendency is intact. The first results of an experiment at our laboratory suggest that these predictions are correct. A third application of the control theory may be to response bias in psychophysical tasks. For example, assimilation and contrast effects in absolute judgments (e.g. Wagner and Baird 1981) might be comparable to the lower order repetition tendencies and repetition avoidance in randomization experiments. While the models used in these studies are formally more sophisticated than those in randomization, the control theory might offer a new way of looking at these data. 


\section{References}

Crowder. R.G. and J. Morton, 1969. Precategorical acoustic storage (PAS). Perception and Psychophysics 5, 365-373.

Evans, F.J. and C. Graham. 1980. Subjective random number generation and attention deployment during acquisition and overlearning of a motor skill. Bulletin of the Psychonomic Society 15. 391-394.

Hays, W.L., 1963. Statistics for psychologists. New York: Ilolt. Rinehart and Winston.

Kuhl. J. and W. Schönpflug, 1974. Ordnungsverhalten, Lärmbelastung und Persönlichkeit. Psychological Research 37, 143-176.

Sanders, A.F., 1975. 'Some remarks on short-term memory'. In: P.M.A. Rabbitt and S. Dornic (eds.), Attention and performance. V. London: Academic Press.

Truijens, C.L., D.A. Trumbo and W.A. Wagenaar, 1976. Amphetamine and barbiturate effects on two tasks performed singly and in combination. Acta Psychologica 40. 233-244.

Tulving, E., 1968. 'Theoretical issues in free recall'. In: T.R. Dixon and D.L. Horton (eds.), Verbal behavior and general behavior theory. Englewood Cliffs. NJ: Prentice-Hall.

Wagenaar, W.A.. 1970. Subjective randomness and the capacity to generate information. Acta Psychologica 22, 233-242.

Wagenaar, W.A.. 1972. Sequential response bias. Doctoral dissertation. Soesterberg. The Netherlands: Institute for Perception TNO.

Wagner, M. and J.B. Baird, 1981. A quantitative analysis of sequential effects with numeric stimuli. Perception and Psychophysics 29, 359-364.

Wiegersma, S., 1982. A control theory of sequential response production. Psychological Research 44. $175-188$. 